\title{
Enfoques y herramientas para la enseñanza de la Termodinámica Técnica durante la pandemia de COVID-19: retos y oportunidades
}

\author{
Begoña Peña ${ }^{a}$, Ignacio Zabalza, Manuel Bailera, Belén Zalba
}

Universidad de Zaragoza - Departamento de Ingeniería Mecánica, 50018 Zaragoza, España. abpp@unizar.es

\section{\$EWWFW}

7КНUDFKIQJ

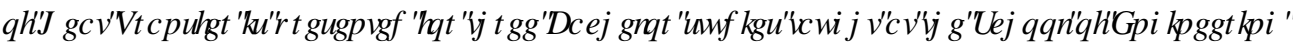

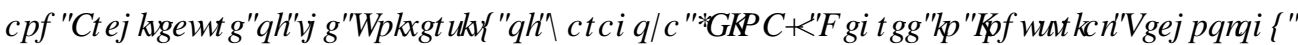

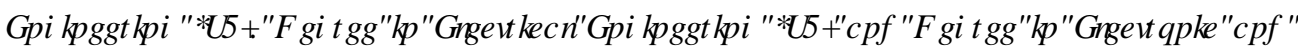

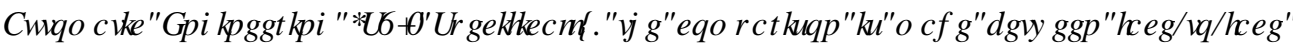

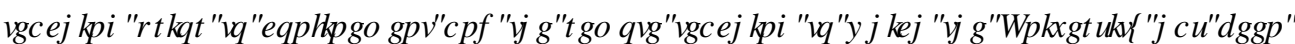

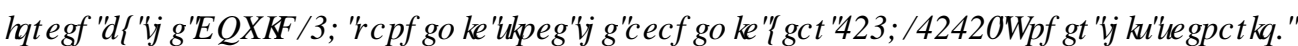

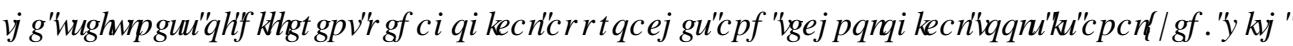

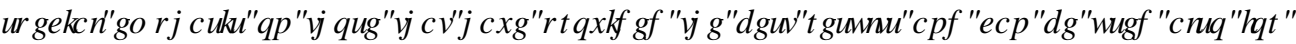
DUADCDQFHUADFKQI

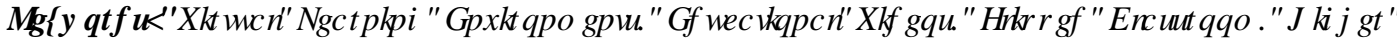

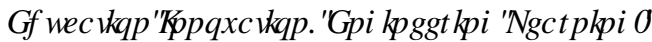

\section{HXP HQD}

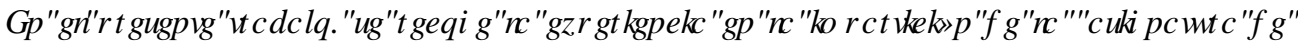

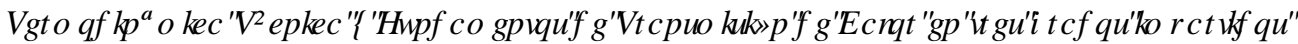

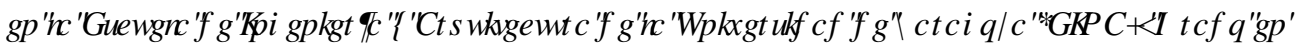

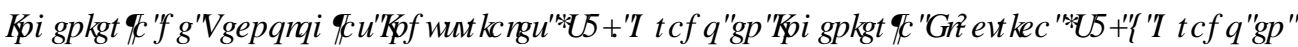

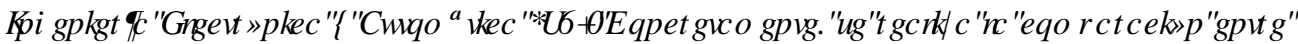

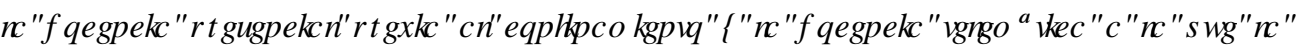

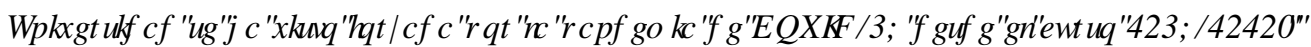

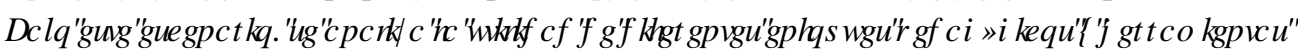
UFQRQ JIFDV KDFIHQRR HSHFIDO PQDUW HQ DTXHODV TXH KDQ SLRSRLFIRQDCR P HRLHV

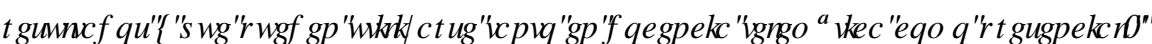

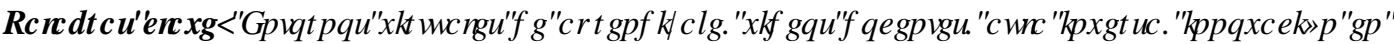
GRFHQHDXQIYHUWUDIDSUHQG] DNAIHQ, QJ HQIHUDD

\section{Introducción}

La pandemia de COVID-19 ha supuesto un gran reto para toda la sociedad en general y para la docencia universitaria en particular. La premura del cambio, la falta de planificación previa y de infraestructura consolidada y las carencias en competencias digitales para adaptarse a la enseñanza a distancia son aspectos diferenciadores con respecto a la enseñanza $R Q Q Q \mathrm{Q} H$ (Hodges, 2020; Nuere, 2020). La necesidad de adaptar en un tiempo record los cursos diseñados para la docencia presencial a los nuevos entornos de enseñanza a distancia ha conllevado un gran esfuerzo por parte de toda la comunidad universitaria y muy especialmente por parte del profesorado. En muchos casos además, las carencias en formación en tecnologías digitales, la 
falta de soporte técnico y la no disponibilidad de equipos adecuados ha dificultado todavía más la labor docente en su objetivo de mantener la motivación de los estudiantes y la calidad del aprendizaje y de realizar una evaluación objetiva y justa (Rapanta, 2020).

Desde el punto de vista del estudiante, se han puesto de manifiesto y se han magnificado las diferencias competenciales, técnicas y económicas en el acceso a medios y herramientas digitales para el seguimiento de la docencia telemática (Coman, 2020; CRUE, 2020; García, 2020). En ciertos entornos, los estudiantes han tenido que enfrentarse a problemas de cobertura, de baja calidad de conexión o a costes excesivos para acceder a Internet (Al-Balas, 2020; Demuyakor, 2020; Mohmmed, 2020. Además, una mejor situación económica se ha constatado que influye positivamente en su grado de motivación y en su estado de ánimo, facilitando el seguimiento de la docencia (Aristovnik, 2020). Por otro lado, ciertos estudios han demostrado que, mediante una buena planificación de actividades, los estudiantes han adquirido hábitos de aprendizaje continuo, mejorando su eficiencia, y han obtenido mejores calificaciones en los exámenes (González, 2020). En este sentido, se observa también que la capacidad para autoregular su aprendizaje influye significativamente en los resultados finales. No obstante, incluso teniendo opiniones positivas sobre la enseñanza telemática, prefieren volver a la enseñanza presencial (EINA, 2020; Rizun, 2020).

A pesar las dificultades, cerca del $70 \%$ de las universidades pusieron en marcha la enseñanza a distancia con apoyo de herramientas digitales desde la primera fase de la pandemia (Marinoni, 2020). A lo largo de 2020, diferentes instituciones elaboraron guías para garantizar la continuidad y la calidad docente (Marinoni, 2020; García, 2020) y mitigar el impacto social y económico sobre la Educación Superior (World Bank, 2020). Las recomendaciones se han orientado hacia: (i) la selección de plataformas y herramientas adecuadas al contexto, minimizando en lo posible su número, (ii) la implementación de medidas para garantizar la conexión de profesores y alumnos, (iii) el apoyo a profesores y alumnos en el uso de herramientas digitales, y (iv) elaborar estrategias de enseñanza, seguimiento y evaluación. En el caso de las Universidades españolas, se han ofrecido cursos de formación, tutoriales y asesoramiento personalizado para el profesorado, se han puesto a disposición del profesorado herramientas para la grabación voluntaria de las clases, se ha facilitado la adaptación de los contenidos y de las metodologías docentes a la modalidad virtual, fomentando la evaluación continua, y se ha utilizado la defensa telemática de trabajos fin de estudios, garantizando la legalidad y la calidad (CRUE, 2020; Mohmmed, 2020; UnizarGuía, 2020).

En la planificación de las actividades de enseñanza-aprendizaje, el profesorado ha tenido que adaptar los contenidos, la metodología de enseñanza-aprendizaje y la evaluación. En este sentido, se ha recomiendado la evaluación continua (García, 2020; González, 2020; Unizar-Guía, 2020) y, en base a la preferencia de los estudiantes, las clases grabadas en video o las clases síncronas mediante videoconferencia (Aristovnik, 2020). Las clases grabadas presentan la ventaja de que pueden verse en cualquier momento, frente a las clases síncronas cuyo seguimiento está sujeto a disponer de una buena conexión de Internet en un horario establecido (Mohmmed, 2020). Por el contrario, las clases síncronas permiten una interacción instántanea que facilita la resolución de dudas en el momento (Coman, 2020).

A pesar de todas las dificultades encontradas, esta situación puede verse como una oportunidad para modernizar y mejorar el aprendizaje en la Universidad. Las competencias digitales adquiridas, los materiales desarrollados para facilitar el seguimiento de la docencia y las estrategias desarrolladas para motivar y favorecer la evaluación continua podrán seguir utilizándose para mejorar y flexibilizar la docencia presencial (EINA, 2020; Quintana, 2020; Sa, 2020)

En el presente trabajo, se recoge la experiencia en la impartición de la asignatura de Termodinámica Técnica y Fundamentos de Transmisión de Calor en tres grados impartidos en la Escuela de Ingeniería y Arquitectura de la Universidad de Zaragoza (EINA): Grado en Ingeniería de Tecnologías Industriales (S3), 
Grado en Ingeniería Eléctrica (S3) y Grado en Ingeniería Electrónica y Automática (S4). Concretamente, se realiza la comparación entre la docencia presencial previa al confinamiento y la docencia telemática a la que la Universidad se ha visto forzada por la pandemia de COVID-19 desde el curso 2019-2020. Bajo este escenario, se analiza la utilidad de diferentes enfoques pedagógicos y herramientas tecnológicas, haciendo especial énfasis en aquellas que han proporcionado mejores resultados y que pueden utilizarse tanto en docencia telemática como presencial.

\section{Objetivos}

En esta comunicación se presentan diferentes enfoques y herramientas utilizados para abordar los retos que presenta la actual situación en la impartición de la asignatura de Termodinámica Técnica y Fundamentos de Transmisión de Calor en tres estudios de grado: Grado en Ingeniería de Tecnologías Industriales (S3), Grado en Ingeniería Eléctrica (S3) y Grado en Ingeniería Electrónica y Automática (S4). El objetivo en todos los casos ha sido aumentar la motivación y la participación de los estudiantes, mantener la calidad del proceso de aprendizaje y la adquisición de competencias y realizar una evaluación objetiva y justa, evitando copia fraudulenta y malas prácticas. Asimismo se reflexiona sobre la posibilidad de aprovechar los materiales y la experiencia ganada para mejorar el aprendizaje bajo docencia presencial en un escenario de normalidad.

\section{Desarrollo de la innovación}

En esta sección se recoge la metodología seguida en las tres asignaturas analizadas. La tabla 1 resume la información general de cada curso, las actividades y metodologías seguidas en cada caso. La nomenclatura utilizada en la tabla para las diferentes titulaciones es la siguiente: GIEA - Grado en Ingeniería Electrónica y Automática, GIE - Grado en Ingeniería Eléctrica, GITI - Grado en Ingeniería en Tecnologías Industriales. Las tres asignaturas son de 6 ECTS y obligatorias de grado en ingeniería. Las dos últimas se imparten en el cuatrimestre de otoño del segundo curso (S3), mientras que la primera se imparte en el cuatrimestre de primavera (S4). Por esta razón, los datos y resultados presentados corresponden al curso 2019-2020 para GIEA y al curso 2020-2021 para GITI y GIE.

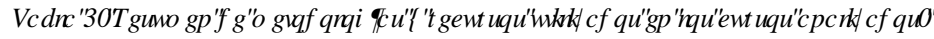

\begin{tabular}{c|c|l|l}
\hline \hline $\begin{array}{c}\text { Grado / } \\
\text { Semestre }\end{array}$ & $\begin{array}{c}\text { Estudiantes } \\
\text { matriculados }\end{array}$ & Metodología y actividades & Recursos \\
\hline GIEA. S4 & 32 & $\begin{array}{l}\text { Clases grabadas en video en canal privado } \\
\text { de YouTube } \\
\text { Google Meet para tutorías y seguimiento }\end{array}$ & $\begin{array}{l}\text { Materiales multimedia } \\
\text { Cuestionarios en Moodle }\end{array}$ \\
\hline GIE. S3 & 59 & $\begin{array}{l}\text { Google Meet para las clases y su chat para } \\
\text { hacer participar a los estudiantes } \\
\text { Aula inversa: Recursos por adelantado, } \\
\text { debate en clase y entrega de tareas breves. }\end{array}$ & $\begin{array}{l}\text { Mapa conceptual } \\
\text { Diario de clase } \\
\text { Foro de noticias de actualidad } \\
\text { Materiales multimedia } \\
\text { Software virtualizado }\end{array}$ \\
\hline GITI. S3 & 56 & $\begin{array}{l}\text { Google Meet y Jamboard para las clases } \\
\text { Aula inversa: Recursos por adelantado, } \\
\text { cuestionarios y entrega de tareas breves. } \\
\text { Dinamización con Mentimeter }\end{array}$ & $\begin{array}{l}\text { Mapa conceptual } \\
\text { Diario de clase } \\
\text { Cuestionarios diversos en Moodle } \\
\text { Materiales multimedia }\end{array}$ \\
\hline \hline
\end{tabular}


Si bien la asignatura tiene la misma denominación en los tres grados, existen diferencias en la dedicación a cada parte. En GITI, el tiempo dedicado a la Termodinámica Técnica es prácticamente el 95\%, incluyendo además el tema de procesos psicrométricos. Por el contrario en GIE y GIEA, la dedicación a la transferencia de calor es mayor, alcanzando prácticamente el 50\%. Esto se debe a que ésta es la única asignatura obligatoria en los respectivos planes de estudios impartida por el área de Máquinas y Motores Térmicos y la transferencia de calor es de gran importancia para la gestión del calor en los sistemas eléctricos y electrónicos.

A pesar de estas diferencias en el contenido, hay que destacar varios puntos comunes a los tres casos de estudio. (i) Se ha seguido haciendo especial hincapié en el uso de los libros de texto recomendados, porque se ha observado un preocupante descenso en su uso en la mayoría de los alumnos en los últimos cursos. El acceso a los libros físicos durante el periodo de confinamiento se ha sustituido por el acceso a los mismos libros en formato digital a través de la plataforma eLibro a la que la Universidad de Zaragoza está suscrita (http://biblioteca.unizar.es/node/1118). (ii) Se han planificado actividades orientadas a favorecer el seguimiento de la evaluación continua. (iii) Se han compartido ideas y recursos. En particular, se han recomendado videos docentes de calidad a través de Moodle o YouTube. La mayoría son videos desarrollados el área de Máquinas y Motores Térmicos de la Universidad de Zaragoza, aunque también se recomiendan algunos de otras universidades. $\square$

\section{Resultados}

En esta sección se detallan los recursos y herramientas utilizados, las actividades realizadas por los estudiantes y el proceso de evaluación seguido, tanto del progreso de los estudiantes como de la metodología y de los recursos proporcionados, a través de encuestas realizadas a los estudiantes.

\subsection{Grado en Ingeniería Electrónica y Automática}

La asignatura analizada se imparte habitualmente de forma presencial durante el cuatrimestre de primavera. Sin embargo, en el curso 2019-2020 la crisis derivada de la pandemia de COVID-19 provocó la cancelación de la enseñanza presencial y su conversión a la enseñanza telemática. Esta circunstancia obligó a la reorganización inmediata de la asignatura desde mitad de marzo hasta el final del curso. Las actividades de enseñanza-aprendizaje se desarrollaron a través de la plataforma Moodle, tratando de seguir un enfoque de aula inversa: proporcionar materiales y aprovechar el tiempo de interacción con los estudiantes para resolver dudas y plantear problemas y casos para su resolución en línea.

Las clases de teoría y problemas se sustituyeron por vídeos desarrollados por el profesor con una duración similar a la de las clases presenciales. En dichos videos, se explican los conceptos teóricos más importantes de la asignatura y se aplican a la resolución de ejercicios prácticos. Los videos de teoría se elaboraron a partir de las presentaciones de PowerPoint con programas de grabación y edición de vídeos. Los videos dedicados a la resolución de problemas se basaron explicaciones del profesor con ayuda de presentaciones que combinaban contenido digital con la resolución manuscrita escaneada. Las presentaciones se distribuyeron a través de Moodle, mientras que se utilizó un canal privado de YouTube para los videos, con el fin de no saturar los servidores de la Universidad de Zaragoza.

El profesor planteó diversas actividades para guiar el aprendizaje: tutorías de seguimiento, cuestionarios de evaluación y entrega de trabajos. Con el fin de monitorizar el progreso de los estudiantes, el profesor supervisó tanto la visualización de los videos como la cumplimentación de los cuestionarios en Moodle.

En cuanto a las sesiones de laboratorio, los estudiantes normalmente resuelven por parejas varios problemas de simulación con la ayuda de diferentes herramientas informáticas. Durante el curso 2019-2020, se adaptó la metodología para su completo desarrollo online. Se mantuvo la programación original en fecha y hora, 
basando su desarrollo en sesiones de chat de texto en Moodle y videoconferencias en Google Meet. En este curso, se contó con el apoyo de un segundo profesor. Durante cada una de las cinco sesiones, el profesor presentó los ejercicios a resolver y permaneció disponible para responder las posibles preguntas. Después de la sesión, los estudiantes enviaron su informe a través de Moodle.

En cuanto a los trabajos tutorados, se mantuvo la planificación original, desarrollándose de forma individual tres tareas guiadas consistentes en ejercicios de tipo examen. Los cuestionarios, informes de laboratorio y ejercicios guiados forman parte de la evaluación continua con un peso total del $35 \%$ de la nota final.

La Figura 1 muestra el número de vistas (acciones pasivas) y mensajes (acciones activas) promedio por alumno matriculado en la asignatura analizada. Como era de esperar, se observa un aumento significativo en ambos casos entre marzo y junio durante los cuales se cancelaron las clases presenciales. Se observan picos en los accesos coincidiendo con las fechas próximas a las tres entregas de los trabajos tutelados (22/04/2020, 24/05/2020 y 05/06/2020), los exámenes parciales $(15 / 05 / 2020$ y $05 / 06 / 2020)$ y el examen final $(17 / 06 / 2020)$.
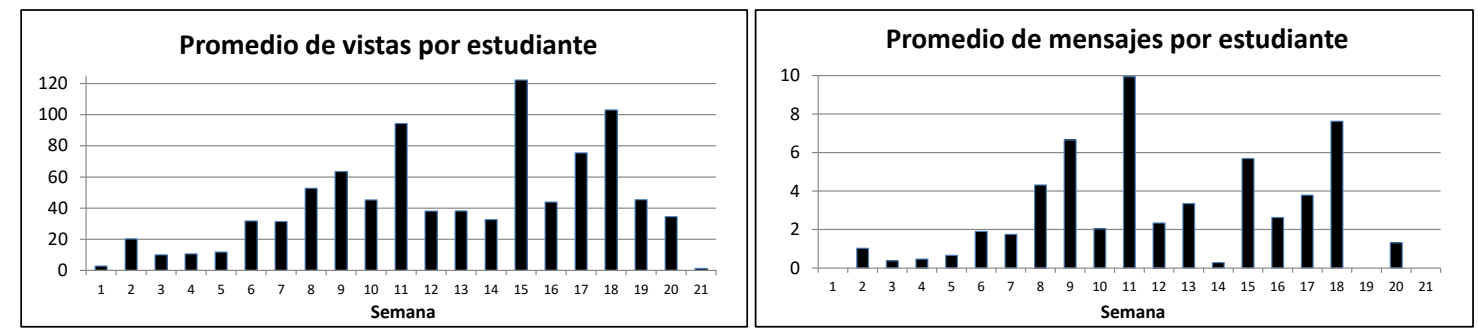

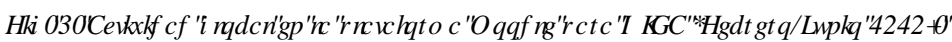

Comparando estas cifras de actividad global con las del curso anterior, tal como cabía esperar, se observa que en el curso 2019-2020 hubo un aumento del 17\% en el número global de vistas y del 16\% en el número total de mensajes.
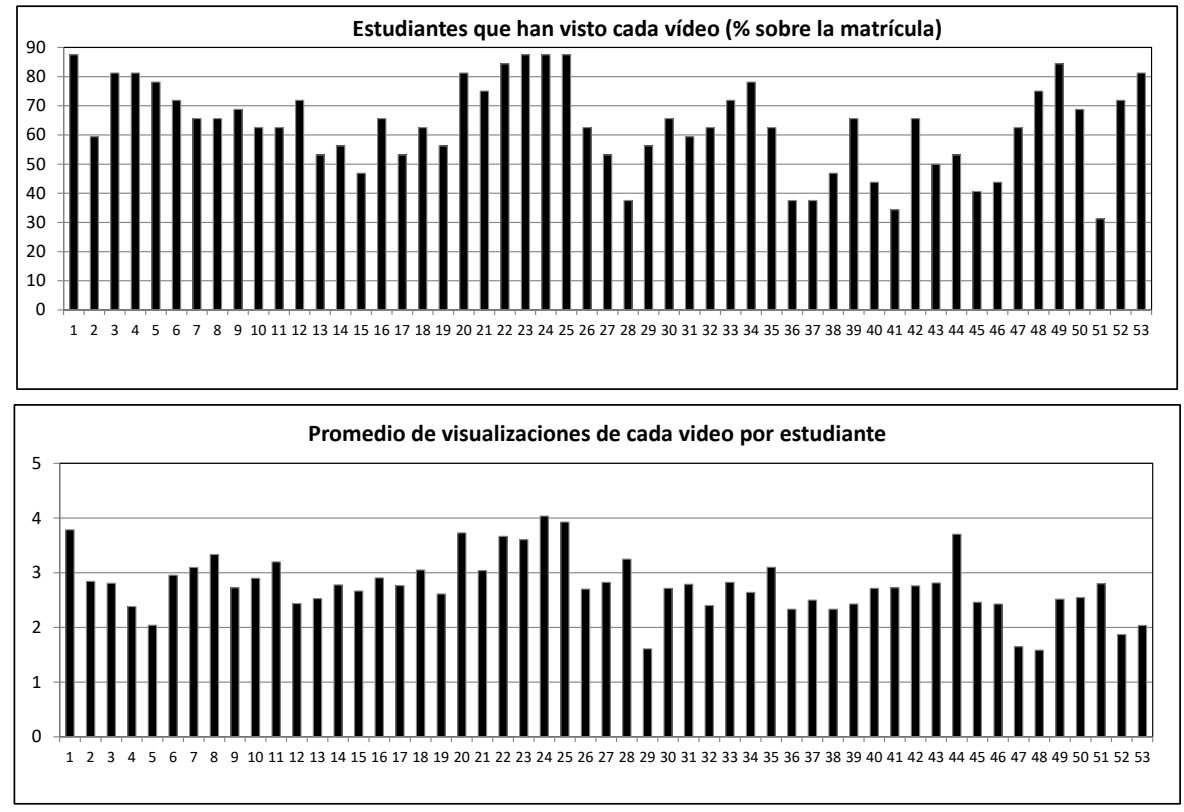

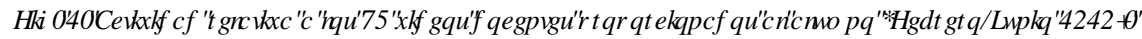

La actividad relacionada con los videos didácticos (Figura 2) muestra el porcentaje de alumnos participantes respecto al número de inscritos y el número de visualizaciones por alumno participante por video. El 
número total de visualizaciones fue ligeramente superior a 3000. El porcentaje de estudiantes que vieron cada vídeo varía del $30 \%$ al $90 \%$, con un valor promedio del $63 \%$. El número de visualizaciones por alumno participante osciló entre 1.6 y 4 , con un valor promedio de 2.8 .

Los delegados del segundo curso de GIE diseñaron y distribuyeron una encuesta para evaluar la enseñanza y los recursos en línea durante el estado de alerta. Ellos mismos recogieron los resultados y los enviaron a los profesores. En una escala Likert del 1 al 5, los recursos aportados en la asignatura analizada fueron evaluados con 4 o 5 para todos los alumnos y el $60 \%$ encontró fácilmente los recursos en la plataforma. Entre los aspectos que más les gustaron, citaron los videos con ejercicios resueltos, los videos teóricos y los cuestionarios que motivaban la visualización de estos videos. Los estudiantes expresaron su preferencia por los videos sobre las videoconferencias, citando tres razones fundamentales: 1) la calidad superior del audio y de la imagen que consideran imprescindible, especialmente en las sesiones donde se explica el uso de herramientas informáticas; 2) la flexibilidad para verlos en cualquier momento; 3) la mejor adaptación a las conexiones de la red doméstica, que son limitadas especialmente cuando hay varias personas trabajando / estudiando en casa (los vídeos se pueden descargar en los momentos en que la conexión es gratuita y/o más rápida). Por otro lado, los estudiantes apreciaron positivamente que se mantuviera la programación de tareas guiadas y las sesiones de laboratorio, ya que les motivó el seguimiento de la evaluación continua, y los ejercicios prácticos que les ayudan a comprender los conceptos y procedimientos teóricos.

En cuanto a las calificaciones, el porcentaje de suspensos bajó ligeramente en comparación con el curso anterior (del 12\% al 9\%). Sin embargo la calificación promedio de la asignatura también bajó (de 7,2 a 6,6) y el porcentaje de no presentados, aún manteniéndose en niveles relativamente bajos, casi se duplicó (del $8 \%$ al $15 \%)$.

\subsection{Grado en Ingeniería Eléctrica}

En el Grado en Ingeniería Eléctrica, la asignatura analizada se imparte normalmente de forma presencial en el cuatrimestre de otoño (S3). Dada la evolución de la pandemia de COVID-19, la Universidad de Zaragoza optó por la docencia telemática en todos los niveles al comienzo del curso 2020-2021, manteniendo presencialidad únicamente para las actividades de evaluación si era posible (Rectorado Unizar, 2020). Por esta razón, la impartición de las clases síncronas se realizó a través de Google Meet y se realizaron diversas adaptaciones para la docencia telemática (Tabla 1).

En el enfoque del curso, se ha tenido muy en cuenta el papel que juegan las emociones en el proceso de aprendizaje. Expertos en neurociencia, como Francisco Mora (Mora, 2003) y Eduardo Bueno (Bueno, 2017) afirman que la emoción es el elemento esencial para fomentar la curiosidad, mantener la atención y activar la memoria durante el aprendizaje. El curso se diseñó y se desarrolló sin perder de vista estas ideas: se han diseñado y utilizado diferentes materiales y actividades y se ha implementado el modelo de aula inversa tanto en la lección introductoria como en diversas actividades prácticas para provocar curiosidad y promover el compromiso de los estudiantes con la asignatura. Las actividades y recursos más interesantes se resumen en la Tabla 2.

Se ha considerado que el inicio del curso es un momento crucial para captar la atención del alumno y redirigirla hacia los contenidos a aprender. Concretamente, el diseño e implementación de diversas actividades durante las primeras semanas del curso 2019-2020 estuvo enfocado precisamente a estos fines. 


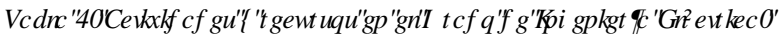

\begin{tabular}{|c|c|c|c|}
\hline $\begin{array}{l}\text { Actividad / } \\
\text { Recurso }\end{array}$ & Descripción & Objetivo & Resultados \\
\hline $\begin{array}{l}\text { Google } \\
\text { Meet }+ \\
\text { PowerPoint } \\
+ \text { cámara }\end{array}$ & $\begin{array}{l}\text { Clases síncronas a distancia, con } \\
\text { cámara de la profesora, } \\
\text { utilizando PowerPoint para } \\
\text { presentaciones y como pizarra } \\
\text { digital }\end{array}$ & $\begin{array}{l}\text { Mantener en lo posible la } \\
\text { planificación y la forma de } \\
\text { impartir el curso }\end{array}$ & $\begin{array}{l}\text { Pocos requerimientos para } \\
\text { adaptar la mayor parte de } \\
\text { los materiales del curso } \\
\text { Clase similar a la presencial }\end{array}$ \\
\hline $\begin{array}{l}\text { Método } \\
\text { socrático } \\
\text { telemático }\end{array}$ & $\begin{array}{l}\text { Se plantean preguntas para que } \\
\text { todos participen, respondiendo a } \\
\text { través del chat de G-Meet de } \\
\text { forma simultánea }\end{array}$ & $\begin{array}{l}\text { Romper el ritmo de la clase } \\
\text { Fomentar la participación } \\
\text { Evaluar el nivel de la clase }\end{array}$ & $\begin{array}{l}\text { Participación muy elevada } \\
\text { Útil para mantener la } \\
\text { atención y para saber el } \\
\text { nivel medio }\end{array}$ \\
\hline $\begin{array}{l}\text { Diario de } \\
\text { clase }\end{array}$ & $\begin{array}{l}\text { Tabla actualizada tras cada } \\
\text { sesión que incluye detalles sobre } \\
\text { materia impartida en cada sesión, } \\
\text { recomendaciones y avisos }\end{array}$ & $\begin{array}{l}\text { Facilitar el seguimiento del } \\
\text { curso, especialmente para los } \\
\text { alumnos que no pueden asistir } \\
\text { de forma síncrona }\end{array}$ & $\begin{array}{l}\text { Valoración muy alta en la } \\
\text { encuesta final } \\
\text { Elevado número de accesos } \\
\text { en Moodle }\end{array}$ \\
\hline $\begin{array}{l}\text { Mapa } \\
\text { conceptual }\end{array}$ & $\begin{array}{l}\text { Resumen y relación entre los } \\
\text { procedimientos que se estudian } \\
\text { en la asignatura }\end{array}$ & $\begin{array}{l}\text { Mantener la visión global del } \\
\text { curso en todo momento }\end{array}$ & $\begin{array}{l}\text { Valoración muy alta en la } \\
\text { encuesta final } \\
\text { Acceso del } 100 \% \text { de los } \\
\text { estudiantes }\end{array}$ \\
\hline $\begin{array}{l}\text { Análisis de } \\
\text { información } \\
\text { actual }\end{array}$ & $\begin{array}{l}\text { Lectura de noticias y búsqueda } \\
\text { de información sobre sistemas } \\
\text { térmicos. Entrega de una } \\
\text { plantilla y autoevaluación de la } \\
\text { misma }\end{array}$ & $\begin{array}{l}\text { Relacionar la asignatura con el } \\
\text { mundo real y con su futuro } \\
\text { desempeño profesional } \\
\text { Fomentar la participación a } \\
\text { través de un debate y de la } \\
\text { coevaluación } \\
\text { Tener retroalimentación del } \\
\text { nivel del grupo y reducir el } \\
\text { tiempo de corrección }\end{array}$ & $\begin{array}{l}\text { Valoración muy alta en la } \\
\text { encuesta final } \\
\text { Clima de confianza } \\
\text { Mayor asistencia y } \\
\text { participación en clases } \\
\text { posteriores }\end{array}$ \\
\hline $\begin{array}{l}\text { Caso: } \\
\text { efecto } \\
\text { Mpemba }\end{array}$ & $\begin{array}{l}\text { En clase se les muestra el } \\
\text { fenómeno y se les da algunas } \\
\text { fuentes de información. Se les } \\
\text { anima a formarse una opinión } \\
\text { crítica para luego hacer un debate } \\
\text { y llegar a ciertas conclusiones }\end{array}$ & $\begin{array}{l}\text { Causar sorpresa y curiosidad } \\
\text { Fomentar la participación a } \\
\text { través de un debate } \\
\text { Introducir cuestiones generales } \\
\text { impotantes para su formación }\end{array}$ & $\begin{array}{l}\text { Valoración muy alta en la } \\
\text { encuesta final } \\
\text { Clima de confianza } \\
\text { Mayor asistencia y } \\
\text { participación en clases } \\
\text { posteriores } \\
\text { Se debe orientar hacia un } \\
\text { aprendizaje }\end{array}$ \\
\hline $\begin{array}{l}\text { Actividades } \\
\text { prácticas } \\
\text { on-line }\end{array}$ & $\begin{array}{l}\text { Utilización de aulas virtuales } \\
\text { para trabajar con software } \\
\text { licenciado } \\
\text { Uso de videos sobre las } \\
\text { instalaciones del laboratorio }\end{array}$ & $\begin{array}{l}\text { Mantener en lo posible la } \\
\text { planificación original } \\
\text { Adaptar o sustituir algunas } \\
\text { visitas al laboratorio }\end{array}$ & $\begin{array}{l}\text { Correcto funcionamiento de } \\
\text { la virtualización del } \\
\text { software } \\
\text { Desarrollo muy similar a la } \\
\text { modalidad convencional }\end{array}$ \\
\hline
\end{tabular}

Por un lado, se anima a los alumnos a leer noticias de actualidad en los periódicos y a buscar información sobre temas particulares relacionados con la asignatura, concretamente sobre el mix de generación eléctrica y el papel de las centrales de ciclo combinado y el almacenamiento de energía en España. El objetivo 
principal de esta actividad es que el alumno relacione la asignatura con el mundo real y con su futuro desempeño profesional. Las tareas guiadas llevan al alumno a darse cuenta de que los ciclos de potencia que se estudian en la asignatura son necesarios para comprender el 50-60\% de los sistemas de generación, almacenamiento y utilización de energía en España. Los estudiantes buscan y analizan la información y completan varias plantillas que son enviadas a través de Moodle y autocorregidas durante una clase dedicada a ello (en el curso 2019-2020 se utilizó coevaluación). Esta estrategia cubre varios objetivos: la autoevaluación es útil para que los estudiantes presten más atención a las explicaciones, a la vez que la profesora obtiene retroalimentación sobre el nivel del grupo y reduce el tiempo de corrección.

El efecto positivo se reflejó en la altísima asistencia a clases observada a lo largo del curso y en las encuestas finales. A pesar de los meses transcurridos desde la actividad, muchos alumnos lo mencionaron en el apartado relacionado con aspectos positivos y puntos fuertes de la asignatura.

La segunda actividad realizada al inicio del curso, basada en el método del caso (Harvard; Yadav, 2010). Fue diseñada en el curso 2019-2020 sobre el llamado efecto Mpemba para causar sorpresa y curiosidad. Esta idea se inspiró en una sesión del evento de divulgación científica 'Pint of Science' impulsado por el investigador Javier Moreno (Universidad de Zaragoza, BIFI) (Moreno, 2019) Este tipo de actividades aplicadas a las clases magistrales también pueden cambiar la actitud de los estudiantes y promover el compromiso (Mora, 2003; Bueno, 2017). De acuerdo con esta idea, la actividad se diseñó e implementó la primera semana del cruso y se aprovechó para introducir algunos contenidos específicos a estudiar en la asignatura, como el equilibrio termodinámico, la inercia térmica o los procesos cuasiestáticos.

Además, esta actividad también se utilizó para introducir hechos generales muy importantes:

- Los fenómenos reales son muy complejos, involucran muchos fenómenos físicos que deben ser investigados desde diferentes áreas de conocimiento, aunque se abordan parcialmente en diferentes materias: "Si algo te parece simple, probablemente no lo estudiaste con suficientemente profundidad".

- Toda medida experimental conlleva incertidumbre: "¿Nuestra precisión y exactitud son suficientes para nuestros propósitos?"

- La información obtenida de Internet puede ser útil y fácil de obtener, pero existen muchas afirmaciones falsas. Como futuros ingenieros, los estudiantes deben utilizar su pensamiento crítico y su autonomía para contrastar fuentes de información y saber encontrar fuentes de conocimiento fiables, como los libros de texto especializados.

Los detalles de esta actividad se pueden encontrar en (Peña, 2020). En opinión de los alumnos, fue una actividad muy interesante y sorprendente. Se involucraron mucho y la participación en el debate fue muy alta. La mayoría de ellos reconoció haber llevado a cabo experimentos caseros en su propio congelador. Esta actividad también se destacó en las encuestas finales como positiva y útil para la asignatura y se recomendó que se repita en los próximos cursos. Desde el punto de vista de la profesora, la actividad despierta el interés de los estudiantes, pero hay que reorientarla de alguna manera hacia un aprendizaje consolidado.

En cuanto a las sesiones de prácticas, debieron realizarse a través de aulas virtuales habilitadas por la EINA para este fin. Dos de las prácticas planificadas en cursos previos consistían en la visita a dos instalaciones: el sistema de climatización del edificio donde estudian y un laboratorio de bomba de calor. En el presente curso, se han sustituido por videos explicativos de dichas instalaciones y la resolución de casos a través del software EES (Engineering Equation Solver). En las prácticas se sigue el modelo de aula inversa: al comienzo de la sesión, la profesora aclara las dudas encontradas para luego pasar a resolver diveros problemas. Las sesiones de laboratorio también fueron mencionadas en las encuestas finales por los estudiantes y desde el punto de vista de la profesora sirven para fomentar la curiosidad por los sistemas de 
calefacción, ventilación y aire acondicionado lo que puede animarles a matricularse en la asignatura optativa relacionada de cuarto curso.

Finalmente, cabe destacar que el éxito en el examen final ha sido similar al del curso anterior, demostrándose así que la docencia telemática no ha supuesto un efecto negativo sobre el proceso de aprendizaje.

\subsection{Grado en Ingeniería de Tecnologías Industriales}

Al igual que para el Grado en Ingeniería Eléctrica, la asignatura analizada se imparte normalmente de forma presencial en el cuatrimestre de otoño (S3), pero durante el curso 2020-2021 la impartición de las clases síncronas se realizó a través de Google Meet, utilizando presentaciones de PowerPoint y Jamboard como pizarra digital. Los recursos y la experiencia en el modelo de aula inversa de cursos anteriores ha servido para que la adaptación docencia telemática sea más rápida y eficiente (Tabla 1).

Algunas de las estrategias como el diario de clase y el mapa conceptual son análogas que para GIE, pero con ciertas diferencias. Concretamente, los campos incluidos en el diario de clase son: materia impartida y documentos asociados, tareas a realizar antes de la próxima clase, materiales recomendados y avisos generales de la asignatura.

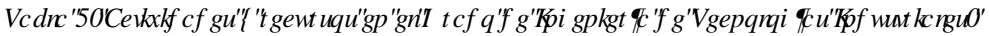

\begin{tabular}{|c|c|c|c|}
\hline $\begin{array}{l}\text { Actividad / } \\
\text { Recurso }\end{array}$ & Descripción & Objetivo & Resultados \\
\hline $\begin{array}{l}\text { Google } \\
\text { Meet }+ \\
\text { PowerPoint } \\
+ \text { Jamboard }\end{array}$ & $\begin{array}{l}\text { Clases síncronas a distancia, } \\
\text { utilizando PowerPoint para las } \\
\text { presentaciones y Jamboard como } \\
\text { pizarra digital }\end{array}$ & $\begin{array}{l}\text { Mantener en lo posible la } \\
\text { planificación y la forma de } \\
\text { impartir el curso }\end{array}$ & $\begin{array}{l}\text { Pocos requerimientos para } \\
\text { adaptar la mayor parte de los } \\
\text { materiales del curso } \\
\text { Clase similar a la presencial }\end{array}$ \\
\hline $\begin{array}{l}\text { Método } \\
\text { socrático } \\
\text { telemático }\end{array}$ & $\begin{array}{l}\text { Se plantean preguntas para que } \\
\text { el alumno participe. Mediante } \\
\text { código QR acceden a la pregunta } \\
\text { en Mentimeter y se discute la } \\
\text { solución }\end{array}$ & $\begin{array}{l}\text { Romper el ritmo de la clase } \\
\text { Fomentar la participación } \\
\text { Evaluar el nivel de la clase }\end{array}$ & $\begin{array}{l}\text { Participación muy elevada } \\
\text { Útil para mantener la } \\
\text { atención y para saber el } \\
\text { nivel medio }\end{array}$ \\
\hline $\begin{array}{l}\text { Diario de } \\
\text { clase }\end{array}$ & $\begin{array}{l}\text { Tabla actualizada tras cada } \\
\text { sesión que incluye detalles sobre } \\
\text { la materia impartida cada día, } \\
\text { tareas a realizar para la próxima } \\
\text { sesión, material recomendado y } \\
\text { avisos }\end{array}$ & $\begin{array}{l}\text { Facilitar el seguimiento del } \\
\text { curso, especialmente para los } \\
\text { alumnos que no pueden asistir } \\
\text { de forma síncrona } \\
\text { Acceso rápido a las tareas y } \\
\text { materiales de aula inversa }\end{array}$ & $\begin{array}{l}\text { Elevado número de accesos } \\
\text { en Moodle }\end{array}$ \\
\hline $\begin{array}{l}\text { Mapa } \\
\text { conceptual }\end{array}$ & $\begin{array}{l}\text { Resumen y relación entre los } \\
\text { procedimientos que se estudian } \\
\text { en la asignatura }\end{array}$ & $\begin{array}{l}\text { Mantener la visión global del } \\
\text { curso en todo momento }\end{array}$ & $\begin{array}{l}\text { Acceso del } 100 \% \text { de los } \\
\text { estudiantes }\end{array}$ \\
\hline $\begin{array}{l}\text { Actividades } \\
\text { prácticas } \\
\text { on-line }\end{array}$ & $\begin{array}{l}\text { Uso de videos para la } \\
\text { preparación previa y } \\
\text { cuestionarios para la evaluación } \\
\text { Uso de presentaciones } \\
\text { PowerPoint interactivas }\end{array}$ & $\begin{array}{l}\text { Mantener en lo posible la } \\
\text { planificación original } \\
\text { Adaptar o sustituir algunas } \\
\text { visitas al laboratorio }\end{array}$ & $\begin{array}{l}\text { Correcto funcionamiento de } \\
\text { la virtualización del } \\
\text { software } \\
\text { Desarrollo muy similar a la } \\
\text { modalidad convencional }\end{array}$ \\
\hline
\end{tabular}


Aunque gran parte del temario se ha impartido bajo el modelo tradicional expositivo, el modelo de aula inversa se ha aplicado como en cursos previos siguiendo un procedimiento similar en las sesiones de laboratorio y en el tema de 3 LRSIHGDGHWGHXWDQFIDVSXWV(Peña, 2020): proporcionar bibliografía, videos y cuestionarios a través de Moodle, dar una breve explicación y resolver dudas al comienzo de la sesión y plantear ejercicios de aplicación. Dado que en modalidad telemática es más difícil el trabajo en grupo y el seguimiento de sus progresos por parte del profesor, este año se ha optado por guiar al alumno en la resolución de los mismos de forma más pautada, corrigiendo cada parte y resolviendo dudas pasados unos pocos minutos. En las sesiones prácticas, la evaluación de la práctica se ha realizado mediante cuestionarios en Moodle con preguntas aleatorias y datos aleatorios, basadas en cálculos similares a los realizados en la práctica. Cabe señalar que las calificaciones de estas actividades correlacionan con la calificación del examen de primera convocatoria con un coeficiente de determinación de 0.57 , lo que quiere decir que esta evaluación es representativa, hasta cierto punto, del aprendizaje del estudiante, dentro de la baja dificultad de estas actividades.

Se ha comparado la actividad global en Moodle para comprender si han cambiado los hábitos de los estudiantes (Moodle, 2013). Las vistas están relacionadas con las actividades pasivas de los usuarios, como accesos, vistas o descargas. Los mensajes incluyen acciones activas del usuario, como la participación en foros, el envío de tareas, la resolución de cuestionarios o encuestas. La Figura 3 muestra las Vistas y Mensajes por estudiante matriculado agrupados por semanas durante el primer semestre (septiembrefebrero) para los dos últimos cursos.

En contra de lo que cabría esperar, no se aprecia un uso muy diferente entre los dos cursos: el número global de vistas fue un $8 \%$ mayor y el número de mensajes un 3\% menor que en el curso 2019-2020. Las mayores diferencias que se observan están relacionadas con las actividades de evaluación. Para el curso anterior los picos en los accesos están asociados a las tres evaluaciones intermedias de los trabajos tutelados $(15 / 10 / 2019,19 / 11 / 2019$ y 17/01/2020) y al examen final (27/01/2020). Durante el presente curso, sólo se realizó una evaluación intermedia (24/11/2020) y el examen final (28/01/2021).
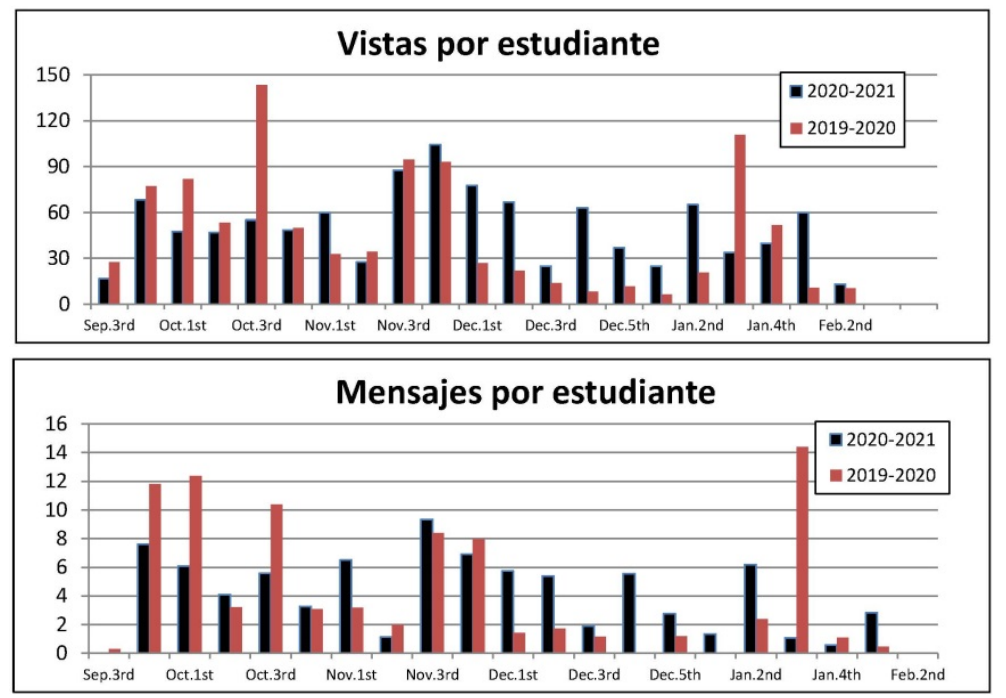

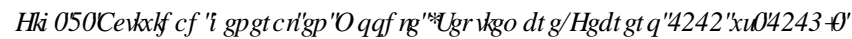

En cuanto al uso de videos, sólo se puede analizar la información relativa a los colgados en Moodle, que se han agrupado por tipología y objetivo en cuatro bloques: recomendados para prácticas, recomendados para el tema 2 impartido bajo el modelo de aula inversa, videos sobre los temas de ciclos termodinámicos de la Universidad Politécnica de Valencia y videos de problemas resueltos en pizarra digital. 
Algunas grabaciones de las clases se compartieron desde una carpeta de Drive para reducir la presión sobre los servidores de la Universidad de Zaragoza, pero no se dispone de los accesos a cada video. En la Figura 4 se compara para los dos últimos cursos el porcentaje de alumnos que vió al menos una vez cada video respecto al número de matriculados y el número de visualizaciones por alumno participante y por video.
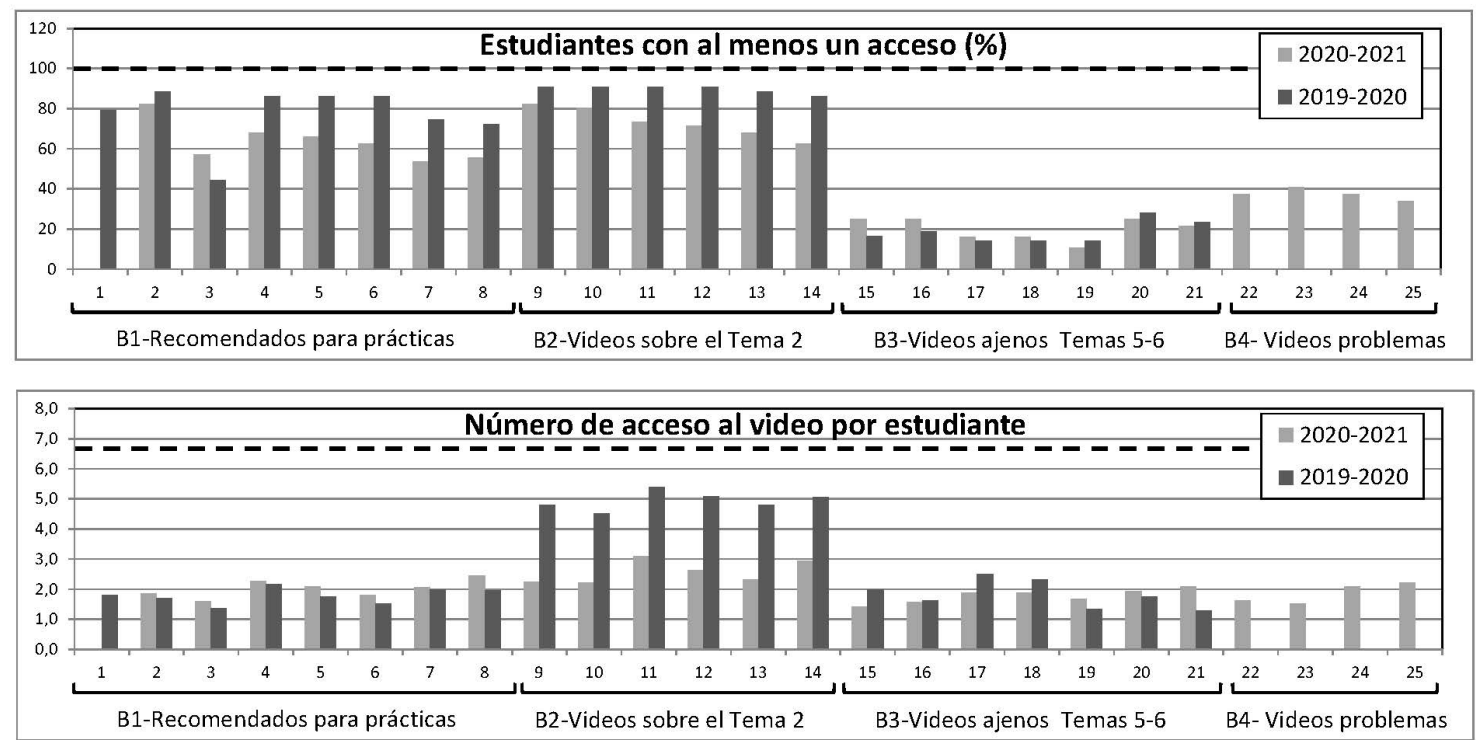

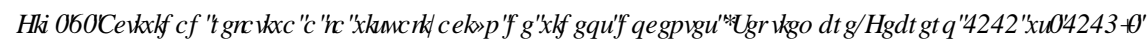

El número total de visualizaciones es superior a 1400 para el curso actual. El porcentaje de estudiantes que vieron cada vídeo varía del $11 \%$ al $82 \%$, con un valor medio del $47 \%$. El número de visualizaciones por alumno participante osciló entre 1 y 3 , con un valor medio de 2.1 .

El porcentaje de alumnos matriculados que siguieron la evaluación continua fue del $75 \%$ frente al $58 \%$ del curso anterior. La tasa de éxito ha aumentado en 4 puntos porcentuales en la primera convocatoria con respecto al curso 2019-2020, a la vez que se ha mantenido prácticamente la nota media de los aprobados: 6.5 durante el curso actual y 6.4 durante el curso previo.

\section{Conclusiones}

Se han presentado tres enfoques diferentes de docencia telemática para la asignatura de Ingeniería Termodinámica y Fundamentos de la Transferencia de Calor en tres Grados de Ingeniería. Las actividades se han diseñado con un objetivo establecido en cada caso según las necesidades observadas por los profesores y en función de los medios disponibles.

En los tres casos de estudio, mantener la motivación y el compromiso ha sido la prioridad a la hora de diseñar los recursos y las actividades. El diario de clase, que tradicionalmente era sólo una herramienta organizativa para el profesor, ha demostrado ser un recurso útil para facilitar el seguimiento de la asignatura por parte del alumno. La herramienta Mentimeter ha sido muy útil en la docencia telématica para conseguir los mismos fines que en docencia en aula: promover la participación, mantener la atención de los estudiantes y proporcionar un feedback inmediato al profesor. El mismo papel ha jugado el uso el chat de Google Meet, utilizado de forma muy ingeniosa en la asignatura del grado de Ingeniería Eléctrica.

En todos los casos, se ha observado una buena acogida por parte de los estudiantes y ello se ha reflejado en un elevado seguimiento de la asignatura en las actividades de evaluación continua. En cuanto al modelo de 
enseñanza, no se ha observado una preferencia especial entre videoconferencias y clases retransmitidas online. Los alumnos se han mostrado conformes con ambas opciones y los resultados de aprendizaje no se ha visto afectados por la adaptación a las clases a distancia, incluso se ha observado un pequeño aumento del número de aprobados en dos de las asignaturas.

Aunque la dedicación al desarrollo de los videos es bastante mayor que la preparación de una clase síncrona (1,5-2 veces), éstos ofrecen algunas ventajas significativas con respecto a las sesiones de videoconferencia: por un lado, son reutilizables en otras asignaturas y otros cursos y, por otro lado, algunos estudiantes los prefieren por su mejor calidad de audio / video, flexibilidad y adaptabilidad temporal.

Finalmente, hay que destacar que la colaboración entre profesores y el intercambio de ideas ha sido muy enriquecedora desde el punto de vista personal y profesional. La colaboración establecida ha supuesto una mejora significativa en las metodologías y recursos didácticos que continuará en el futuro.

\section{Agradecimientos}

Este trabajo se ha desarrollado en el marco del proyecto PIIDUZ_19_265 (Programa de Proyectos de Innovación Docente para Grupos de Profesores del Vicerrectorado de Política Académica de la Universidad de Zaragoza). El proyecto ha sido coordinado por la profesora Begoña Peña Pellicer, siendo los profesores participantes: Ignacio Zabalza Bribián, Manuel Bailera Martín, Eva $\mathrm{M}^{\mathrm{a}}$ Llera Sastresa, Enrique Teruel Doñate, Sergio Usón Gil, Ma Belén Zalba Nonay, Pilar Lisbona Martín, Ana Iris Escudero Oriol, Sara Pascual Sevilla, María Aznar Montesinos y Gabriel Peris Millán. Los autores agradecen su contribución al proyecto.

\section{Referencias}

AL-BALAS ET AL. (2020) "Distance learning in clinical medical education amid COVID-19 pandemic in Jordan: current situation, challenges, and perspectives". BMC Medical Education 20:341, 2020. https://doi.org/10.1186/s12909-020-02257-4.

AREA MMT EINA-UZ (2021). "Innovación Docente Area MMT EINA-UZ”. Youtube channel of Thermal Machines and Engines area of the University of Zaragoza. https://www.youtube.com/channel/UCrnGX5EreK1Ot06-XswAV3Q. (Último acceso: 29/03/2021).

ARISTOVNIK A., KERŽI`C D., RAVŠELJ D., TOMAŽEVIC N. Y UMEK L.(2020) "Impacts of the COVID-19 Pandemic on Life of Higher Education Students: A Global Perspective". Sustainability 2020, 12, 8438; doi:10.3390/su12208438.

BUENO, D. (2017), "Neurociencia para Educadores". Ediciones Octaedro, Barcelona, 2017. ISBN: 97884-9921-991-2.

BURRIDGE, H.C., y LINDEN, P.F. (2016) “Questioning the Mpemba effect: hot water does not cool more quickly than cold”, Nature, Scientific Reports, vol. 6, pp. 37665, 2016. DOI: 10.1038/srep37665.

CRUE (2020) "La Universidad frente a la pandemia". Conferencia de Rectores de las Universidades Españolas. Disponible en: https://www.crue.org/2020/12/universidad-frente-pandemia/ (Último acceso: 29/05/2021).

DEMUYAKOR, J. (2020) "Coronavirus (COVID-19) and Online Learning in Higher Institutions of Education: A Survey of the Perceptions of Ghanaian International Students in China". Online Journal of Communication and Media Technologies, 10(3), e202018. https://doi.org/10.29333/ojcmt/8286. 
EINA (2020) "La evaluación no presencial en la EINA: supervivencia u oportunidad”. Jornadas virtuales, 4 y 15 de Julio de 2020, Escuela de Ingeniería y Arquitectura, Universidad de Zaragoza. https://eina.unizar.es/noticias/documentos-de-la-jornada-virtual-la-evaluacion-no-presencial-en-la-einasupervivencia-u (Último acceso: 29/05/2021).

GALINDO J., Canal de YouTube "Universitat Politècnica de València - UPV". https://www.youtube. com/channel/UCsANOtTfEeqqFMkyRnfCnOQ. (Último acceso: 29/03/2021).

GARCÍA-PEÑALVO F.J., CORELL A., ABELLA-GARCÍA V. y GRANDE M. (2020) "Online Assessment in Higher Education in the Time of COVID-19". Education in the Knowledge Society 21 (2020) article 12. Ediciones Universidad de Salamanca. https://doi.org/10.14201/eks.23013.

GONZALEZ T, DE LA RUBIA MA, HINCZ KP, COMAS-LOPEZ M, SUBIRATS L, FORT S, ET AL. (2020) "Influence of COVID-19 confinement on students' performance in higher education". PLoS ONE 15(10): e0239490. https://doi.org/10.1371/journal.pone.0239490.

HARVARD BUSINESS SCHOOL. "The HBS case method". https://www.hbs.edu/mba/academicexperience/Pages/the-hbs-case-method.aspx. (Último acceso: 29/03/2021).

HODGES C., Moore S., Lockee B., Trust T. y Bond A. (2020) "The Difference Between Emergency Remote Teaching and Online Learning". Educause Review. https://er.educause.edu/articles/2020/3/thedifference-between-emergency-remote-teaching-and-online-learning (Último acceso: 29/05/2021)

MARINONI, G., VAN'T LAND, H.; y JENSEN, T. (2020) “The Impact of Covid-19 on Higher Education Around the World". IAU Global Survey Report, mayo 2020. Disponible en: https://www.iauaiu.net/IMG/pdf/iau_covid19_and_he_survey_report_final_may_2020.pdf (Último acceso: 29/05/2021)

MOHMMED AO., KHIDHIR B.A., NAZEER A. Y VIJAYAN V.J. (2020) "Emergency remote teaching during Coronavirus pandemic: the current trend and future directive at Middle East College Oman". Innovative Infrastructure Solutions (2020) 5:72. https://doi.org/10.1007/s41062-020-00326-7.

MOODLE (2013). Moodle.org. Course overview report, 2013. Manual online, disponible en: https://docs.moodle.org/25/en/Course_overview_report. (Último acceso: 29/03/2021).

MORA. F. (2003). “Neuroeducación”. Alianza Editorial, Madrid, 2003. ISBN: 978-84-9104-780-3.

MORENO, J. (2019) "Efecto Mpemba. Haciendo helados". Pint of Science. Teatro de las Esquinas, Zaragoza (Spain). May 2019.

NUERE S. y DE MIGUEL L. (2020) “The Digital/Technological Connection with COVID-19: An Unprecedented Challenge in University Teaching". Technology, Knowledge and Learning https://doi.org/10.1007/s10758-020-09454-6.

PEÑA, B., BAILERA, M., ZABALZA, I. y ZALBA, B. (2010). "The flipped classroom model in engineering thermodynamics: comparison of experiences in different bachelor degrees" en $\square \mathrm{WK}$

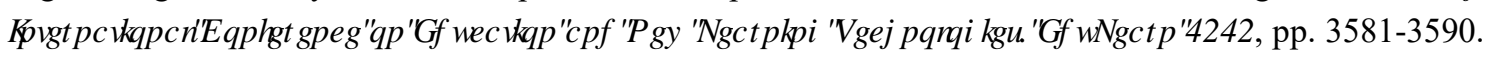
http://dx.doi.org/ 10.21125/edulearn.2020.0999.

QUINTANA R. y QUINTANA C. (2020) "When classroom interactions have to go online: the move to specifications grading in a project-based design course". Information and Learning Sciences Vol. 121 No. 7/8, 2020 pp. 525-532. https://doi.org/10.1108/ILS-04-2020-0119.

RAPANTA, C., BOtTURI, L., GOODYEAR, P., GUÀRdiA, L. Y KOOLE, M. (2020). “Online University Teaching During and After the Covid-19 Crisis: Refocusing Teacher Presence and Learning 
Activity". 3RWUJWDO6FHQFHDQG) ( GXFDMRQ vol 2, pp. 923-945. https://doi.org/10.1007/s42438-02000155-y.

RECTORADO UNIZAR (2020). "Resolución del Rector en funciones de la Universidad de Zaragoza, de 24 de septiembre de 2020, por la que se aprueban instrucciones aplicativas referidas al Acuerdo del Consejo de Gobierno de 6 de julio de 2020, por el que se adoptan directrices para afrontar el desarrollo de la docencia en el curso académico 2020-21, en la situación de nueva normalidad, así como algunas especificaciones en materia de prevención" 24/09/2020. https://www.unizar.es/covid. (Último acceso: 29/05/2021)

RIZUN M. y STRZELECKI A. (2020). "Students' Acceptance of the COVID-19 Impact on Shifting Higher Education to Distance Learning in Poland". Int. J. Environ. Res. Public Health 2020, 17, 6468; doi:10.3390/ijerph17186468.

Sá M.J. y Serpa S. "The COVID-19 Pandemic as an Opportunity to Foster the Sustainable Development of Teaching in Higher Education”. Sustainability 2020, 12, 8525; doi:10.3390/su12208525.

UNIVERSIDAD DE SEVILLA (2021). ¿En qué consiste el efecto Mpemba?” Youtube channel of Sevilla University. https://www.youtube.com/watch?v=FRpAaCwD-18. (Último acceso: 29/03/2021).

UNIVERSIDAD DE ZARAGOZA (2021). OCW course "Material audiovisual de apoyo al estudio de la termodinámica y la ingeniería térmica". University of Zaragoza. https://ocw.unizar.es/ocw/mod/resource/view.php?id=666\& forceview=1. (Último acceso: 29/03/2021).

UNIZAR-ADD (2020). "Herramientas evaluación online Unizar- COVID19”. Universidad de Zaragoza. https://add.unizar.es/add/campusvirtual/herramientas-evaluacion-online-unizar (Último acceso: 29/05/2021).

UNIZAR-GUIA (2020). "Guía de la Universidad de Zaragoza para la adaptación a la docencia no presencial y evaluación online". Aprobada en Consejo de Gobierno de 27 de abril de 2020. https://internacional.unizar.es/sites/internacional.unizar.es/files/archivos/pdf/covid/guia_adaptacion_evalu acion_no_presencial_cg20200420.pdf (Último acceso: 29/05/2021).

WORLD BANK (2020) “The COVID-19 Crisis Response: Supporting Tertiary Education for Continuity, Adaptation, and Innovation". World Bank, Washington, DC. (C) World Bank. https://openknowledge.worldbank.org/handle/10986/34571

YADAV, A., SHAVER, G.M. y MECKL, P. (2010). "Lessons Learned: Implementing the Case Teaching Method in a Mechanical Engineering Course". Journal of Engineering Education, vol 99, pp. 55-69, 2010. 